\title{
LA EVOLUCION DEL REGIMEN DE DISCIPLINA EN EL NIVEL MEDIO DE ENSEÑANZA. CIUDAD DE BUENOS AIRES, ARGENTINA (1943-2010)
}

\author{
Natalia Alvarez Prieto (UBA-CmEICS)
}

\section{Resumen}

En este artículo analizaremos la evolución del régimen disciplinario argentino correspondiente al nivel medio de enseñanza, desde la década del ' 40 hasta la actualidad, a partir del estudio de la normativa legal. Asimismo, estudiaremos las transformaciones operadas en una jurisdicción en particular: la Ciudad de Buenos Aires. Allí, hacia fines de los años '90, se introdujo una reforma que modificó sustancialmente el régimen disciplinario existente: la creación del Sistema Escolar de Convivencia. Dicha reforma constituyó un caso testigo que, luego, se fue replicando en diversas jurisdicciones. Por ello, resulta de interés analizar las características de lo que, tal vez, haya sido un primer momento de una reforma del conjunto del sistema educativo argentino.

Para dar cuenta de nuestro objetivo, examinaremos la legislación nacional y jurisdiccional, correspondientes al período bajo estudio. Asimismo, consideraremos diversos documentos históricos tales como los informes y diagnósticos sobre el sistema educativo efectuados por funcionarios y especialistas de la educación.

\section{Palabras clave}

Sistema disciplinario - Nivel medio - Normativa legal - Reforma educativa - Sistema escolar de convivencia.

\section{Abstract}

This article analyzes the evolution of the argentine discipline system for the middle level of education, from the '40s to the present, based on the study of legal norms.

We will also study the transformations in a particular jurisdiction: the City of Buenos Aires. In the late 90s, there was created the Coexistence School System, a reform that substantially altered the existing disciplinary rules. This reform was a test case

Licenciada en Sociología. Facultad de Ciencias Sociales. Universidad de Buenos Aires. Becaria Doctoral del CONICET. 
that later was replicated in various jurisdictions. It is therefore of interest to analyze the characteristics of what perhaps was a first step to a reform of the entire educational system in Argentina.

To aim our objective, we will examine national and juridisctional legislation for the period under study. Also, we will consider various historical documents such as reports and assessments of the education system made by officials and education specialists.

\section{Key Words}

Discipline system - Middle level - Legal norms - Educational reform - Coexistence school system.

\section{Introducción}

En este artículo nos proponemos rastrear la evolución del régimen disciplinario argentino correspondiente al nivel medio de enseñanza, desde la década del '40 hasta la actualidad, a partir del estudio de la normativa legal. Asimismo, analizaremos las transformaciones operadas en una jurisdicción en particular: la Ciudad de Buenos Aires. Allí, hacia fines de los años '90, se introdujo una reforma que modificó sustancialmente el régimen disciplinario existente: la creación del Sistema Escolar de Convivencia. Cabe destacar que dicha reforma constituyó un caso testigo que, luego, se fue replicando en diversas jurisdicciones. De hecho, la Ley de Educación Nacional, sancionada en el año 2006, ha retomado algunos elementos introducidos previamente en la experiencia desarrollada en la Ciudad, tales como la participación de docentes, familias y estudiantes en diversos ámbitos de la vida de las instituciones educativas, y los ha generalizado para el conjunto educativo. Asimismo, recientemente, comenzaron a delinearse diversos proyectos legislativos que se proponen extender la experiencia de la Ciudad a diversas provincias e, incluso, al conjunto del país. Por ello, consideramos que resulta de interés analizar las características de lo que -en una perspectiva de largo plazo- tal vez haya sido un primer momento de una reforma del conjunto del sistema educativo argentino.

Para dar cuenta de nuestro objetivo, examinaremos la legislación nacional y jurisdiccional, correspondientes al período bajo estudio. Asimismo, consideraremos diversos documentos históricos tales como los informes y diagnósticos sobre el sistema educativo efectuados por funcionarios y especialistas de la educación.

Proponemos historizar la disciplina en el nivel medio estructurando el relato a partir de los distintos momentos de quiebre o ruptura, aún cuando éstos no hayan cuajado en la normativa. De ese modo, en el primer acápite nos detendremos en el análisis del régimen de disciplina vigente durante casi 
sesenta años. En el segundo, estudiaremos las reformas introducidas por la última dictadura militar orientadas a reforzar, en el espacio educativo, las tareas políticas más generales que llevó a cabo. A su vez, veremos cómo para el régimen dictatorial no resultó apremiante reestructurar la disciplina escolar en el plano normativo. En el tercer acápite, analizaremos la emergencia, a partir de la reapertura democrática de 1983, de una tendencia cuyas notas centrales fueron el reclamo de una escuela más "participativa" y "democrática". En cuarto lugar, estudiaremos las innovaciones que introdujo el Sistema Escolar de Convivencia hacia fines de los años '90. Por último, propondremos algunas claves de lectura para comprender la evolución del sistema disciplinario en el período bajo estudio (1943-2010).

\section{Una breve historia de la normativa: \\ el "sistema de amonestaciones" de 1943}

En marzo de 1943 el Ministerio de Justicia e Instrucción Pública encomendó a la Dirección General de Instrucción Pública, en carácter de urgente, la redacción de un proyecto de Reglamento General para los establecimientos de enseñanza media (Resol. 29/03/1943). De ese modo, se buscaba unificar la multiplicidad de reglamentos y disposiciones vigentes hasta ese momento. Según el Director General de Instrucción Pública, existían más de 1.550 artículos de distintos reglamentos, 600 decretos y resoluciones y un millar de circulares del Ministerio y de la Inspección General de Enseñanza, configurando una elevada dispersión de la normativa (Dirección General de Instrucción Pública, 1943). En forma expeditiva, el proyecto fue presentado y aprobado en mayo de ese mismo año.

Así, un primer momento de nuestro recorrido lo constituye la sanción del Reglamento General para los establecimientos de enseñanza secundaria, normal y especial en 1943. Allí, se establecieron las normas disciplinarias que debían regir, a nivel nacional, en el nivel medio de enseñanza. Cabe destacar que aquella normativa, conocida como el "sistema de amonestaciones", pervivió -con algunas modificaciones- durante poco más de medio siglo en todo el país. Resulta central, entonces, examinarla.

Todo código legal parte determinando los deberes y derechos de los sujetos sobre los que legisla. El Reglamento General no fue la excepción y se encargó de sentarlos. Los deberes de los alumnos se establecieron de manera taxativa en relación a aspectos tales como la obligación de respetar a sus superiores, observar una buena conducta dentro y fuera del establecimiento y conducirse en las clases con aplicación y cultura, entre otros. En lo que hacía a las prohibiciones, se vetaba la posibilidad de ingresar a aulas distintas a las asignadas, tomar parte en actos de indisciplina colectiva, llevar al establecimiento libros y útiles en exceso o libros y papeles que no tuvieran relación con los estudios y exhibir insignias, emblemas o distintivos lesivos a la institución o a las autorida- 
des del Estado. Cabe señalar que los actos de indisciplina colectiva implicaban la expulsión inmediata -por un año o más según la gravedad del hecho. En otro nivel, se buscaba inculcar, además, el cuidado del patrimonio escolar. De ese modo, en el caso de roturas o daños efectuados a los bienes de la institución, más allá de las medidas disciplinarias dispuestas, se imponía su reparación por parte de los alumnos involucrados, quienes no podían volver al establecimiento hasta tanto efectuaran tal resarcimiento.

El Reglamento no sólo pautó aspectos tan generales sino que también se encargó de jerarquizar las sanciones aplicables por mala conducta. Éstas eran, en orden de importancia, la/s amonestación/es, la separación temporal del establecimiento, la expulsión y, llegado el caso, la expulsión de todos los establecimientos de la República. En relación a las amonestaciones, debían aplicarse, según el criterio del Rector o Director, en número proporcional a la falta cometida. La sucesiva acumulación de amonestaciones derivaba en la pérdida de la regularidad por parte del alumno, situación a la que se llegaba al sumar un total de veinticinco. No obstante, el reglamento contemplaba una excepción: el alumno podía solicitar un margen de cinco amonestaciones más. Era el Rector o Director quien, luego de verificar los antecedentes del estudiante y de observar que éste no registrase ningún aplazo en sus promedios, debía resolver dicha solicitud.

Al mismo tiempo que normaba las sanciones, el Reglamento indicaba que éstas debían ser aplicadas cuidadosamente. En ese sentido, señalaba que no podían ser el efecto indiscriminado de un movimiento de "mal humor" sino el resultado de una decisión adoptada con justa reflexión, aunque con "sentimiento y afectuosa gravedad", en procura de enmienda y corrección. Asimismo, alertaba sobre la evasión, por parte de los docentes y celadores, de la autoridad competente de aplicación de las sanciones en pos de criterios arbitrarios:

Deberá cesar la práctica antirreglamentaria de que los profesores y celadores pidan castigos determinados y precisen el número de amonestaciones por aplicarse, en vez de limitarse a señalar, con las circunstancias del caso, la falta que debe corregirse a la única autoridad encargada de discriminar y resolver. (p. 161)

En esa dirección, también se encargaba de fijar los criterios que serían utilizados en relación a una de las máximas sanciones: el ostracismo educativo. Así, la separación temporal del establecimiento sólo podía ser aplicada por el Rectorado o Dirección por un lapso que no superase un año escolar. En caso de requerirse un término mayor, se pronunciaría el Consejo de Profesores del curso al que pertenecía el alumno sancionado. Por su parte, la expulsión sólo podía ser resuelta por aquel Consejo, convocado y presidido por el Rector o Director, al que debía asistir, como mínimo, los dos tercios de su totalidad. Allí también se determinaría si la expulsión comprendería a todos los establecimien- 
tos. En caso de que así fuera, la medida debía ser sometida a la aprobación de la Dirección General de Enseñanza. Además de esos mecanismos, el Reglamento garantizaba que el alumno fuera oído antes de ser juzgado. Finalmente, la expulsión se dictaría con los votos de, por lo menos, las dos terceras partes de los profesores miembros del Consejo. La expulsión implicaba la pérdida de la condición de regular del alumno en tanto quedaba inhabilitado a continuar sus estudios en otro establecimiento durante el curso escolar. Entre las causas de expulsión definitiva figuraban faltas tales como la "inmoralidad grave", el menoscabo reiterado del respeto a profesores o autoridades, una notoria mala conducta dentro o fuera del establecimiento, así como todas las que el Consejo de Profesores considerase.

Finalmente, el Reglamento determinaba que la calificación de la conducta constaría en el boletín de calificaciones del alumno. En ese sentido, ésta se evaluaba en cada período lectivo con las apreciaciones de buena (sin amonestaciones), regular (hasta diez amonestaciones), deficiente (hasta veinte amonestaciones) y mala (más de veinte amonestaciones).

Como hemos dicho, hasta fines de la década del noventa, la normativa fijada en 1943 no sufrió cambios sustanciales. Sin embargo, creemos que reviste sumo interés analizar, con algo de detalle, las políticas implementadas durante la última dictadura militar en relación a la disciplina escolar. A ello nos abocaremos en el próximo acápite.

\section{La disciplina patriótica}

La última dictadura militar llevó a cabo numerosas reformas en el conjunto del sistema educativo. En relación al tema que nos ocupa, en el año 1979 fue constituido un grupo de trabajo encargado de preparar un anteproyecto de Reglamento General para el nivel medio (Resolución No 1.534). De esa forma, el 24 de marzo de 1981, por Resolución Ministerial № 413, se sancionó un Reglamento Orgánico para el Nivel Medio. En lo que refiere a la disciplina, el nuevo reglamento introducía muy pocas modificaciones. Entre ellas, se incorporaba el deber de respeto a los símbolos patrios y la prohibición de colocar carteles o asentar leyendas en paredes y muebles. En cuanto a las sanciones, se introdujo el apercibimiento y, como causa de expulsión, el no respetar y honrar a los símbolos patrios. Sin embargo, la nueva normativa tuvo vigencia durante sólo dos meses. El argumento esgrimido fue que el Reglamento no había considerado diversos aspectos del quehacer escolar, por lo que sería suspendido hasta tanto se completara su redacción (Resolución Ministerial $\mathrm{N}^{\circ}$ $225 / 81$ ). Ahora bien, ¿cuáles fueron las notas centrales de los escasos cambios que intentaron introducirse en el régimen de disciplina?

El respeto hacia los símbolos patrios no sólo se impuso como un deber de los alumnos, sino, también, como una de las funciones principales e indelegables de los profesores. Es decir, los docentes debían despertar y desarrollar en los 
estudiantes el amor a la Patria, el acatamiento de la Constitución Nacional y las leyes, la defensa de los derechos soberanos sobre el territorio nacional y el respeto por la tradición y el patrimonio cultural de la Nación. En ese sentido, en el año 1978 habían sido establecidas las "Normas sobre las características, tratamiento y uso de los símbolos nacionales" (Resolución Ministerial № 1.635). Los especialistas partían del siguiente diagnóstico:

La institución educativa ha sido alcanzada y afectada por la prédica y el accionar de nefastas tendencias ideológicas, cuyo objetivo es la destrucción progresiva de los principios y valores que sustentan y definen la argentinidad, con el propósito de lograr su aniquilamiento y sustitución por concepciones despersonalizantes, materialistas y antinacionales. (Boletín de Comunicaciones $N^{\circ} 61,1978$, p. 29)

A la luz de aquella caracterización, señalaban la necesidad de que los docentes fueran modelo de patriotismo, así como su obligación de exigir a los alumnos la respuesta "debida" ante los símbolos patrios. De esa forma, la docencia debía convertirse en prédica y acción formativa patriótica permanente. Ya en su discurso de asunción, el 30 de marzo de 1976, Ricardo Pedro Bruera sostuvo que la labor de la etapa era "reintegrar a la escuela argentina dentro del sentido nacional y de sus funciones al servicio del país" (p. 6). Asimismo, la escuela secundaria debía promover la formación de hábitos morales y religiosos y el desarrollo de actitudes cívico-sociales.

Si bien el régimen castrense tuvo como uno de sus objetivos reformular la normativa que regulaba la vida de las instituciones educativas del nivel medio, evidentemente, no fue ésta una de sus prioridades. Sólo así puede entenderse que durante aquella etapa no haya podido cuajar ninguna reforma sustantiva en ese sentido. Ahora bien, ¿por qué no fue una prioridad transformar el régimen disciplinario? Desde nuestra perspectiva, dos elementos centrales permiten explicarlo: el carácter represivo del sistema disciplinario tradicional y la clandestinidad en la cual se ejerció la represión.

En primer lugar, el Reglamento que regía la vida de los establecimientos de enseñanza media tuvo una fuerte impronta represiva desde su misma configuración, en 1943. Ejemplo de ello fue la prohibición de la organización colectiva de los estudiantes. Así, el artículo $n^{\circ} 173$ estableció que las autoridades educativas de los establecimientos no atenderían, bajo ningún concepto, representaciones colectivas de los alumnos, ni por sí ni por "los llamados centros o entidades estudiantiles". Por otro lado, se trataba de una normativa sumamente ambigua en relación a un punto central: la expulsión de los alumnos, que podía encontrar su origen en una rateada grupal o en una toma de colegio. En ese sentido, permitía su adecuación a los intereses políticos dominantes de cada momento histórico. De ese modo, creemos que la ambigüedad del régimen disciplinario resultó funcional a las tareas que se propuso la última dictadura en el ámbito 
educativo: en palabras de los funcionarios, su "despolitización" y "depuración ideológica". Términos que, en realidad, ocultaban la eliminación de la fuerza social revolucionaria ("despolitización") y la naturalización del orden establecido ("depuración ideológica"). Precisamente, en el contexto social y político más general, la dictadura se proponía saldar, por un lado, la brecha hegemónica abierta luego de la caída de Perón en 1955 y, por el otro, regimentar a las clases a partir de la eliminación de una fuerza social -la revolucionaria- emergida luego del ascenso de masas conocido como el "Cordobazo" en 1969 (Sartelli, 2007).

En segundo lugar, en tanto el objetivo central de la dictadura fue sentar las bases para reconstruir la hegemonía burguesa, la disciplina escolar se dirimió, fundamentalmente, en el terreno de la lucha de clases. Por ello, para el régimen castrense resultaba apremiante la eliminación de la organización estudiantil. Como hemos visto, las herramientas normativas para desplegar aquella tarea ya habían sido establecidas treinta años antes. Sin embargo, más importante aún fue el hecho de que el disciplinamiento social se llevó a cabo, también en el espacio educativo, a partir del accionar represivo-clandestino del Estado. Realidad que afectó a estudiantes y docentes (Pineau, 2006).

En suma, la transformación del código disciplinar correspondiente al nivel medio resultó, si no innecesaria, por lo menos, no apremiante. Creemos que es por ello que el viejo código legal de 1943 permaneció inmutable en un momento crucial de la historia argentina.

\section{"Participación vs. autoritarismo"}

Luego de la última dictadura militar, comenzaron a introducirse ciertas reformas reglamentarias que anticiparían una nueva etapa. En ese sentido, el Decreto $n^{\circ} 898$, sancionado en 1984 , le dio basamento a las transformaciones que se buscaban iniciar en tanto delegó al Ministro de Educación y Justicia la facultad de modificar y sustituir el arcaico reglamento disciplinar vigente. Uno de los puntos nodales que la "era democrática" buscaba revertir era los vestigios de autoritarismo en toda la normativa del sistema educativo. En un sentido más amplio, podemos afirmar que los cambios en el régimen disciplinar se enmarcaron dentro de una tarea más vasta de revisión del funcionamiento del sistema educativo. Para ello, el gobierno radical fijó toda una agenda de debate y discusión educativa en esos años. El arquetipo de esas intenciones fue la convocatoria a un Congreso Pedagógico Nacional para debatir sobre las posibles transformaciones de la educación en su conjunto. Escapa a las intenciones de este artículo el examen de aquella experiencia. Sin embargo, debemos tenerla en cuenta a la hora de contextualizar la serie de cambios en el régimen disciplinar que aquí estudiaremos.

Avanzando en esa tarea de innovación, en el año 1985 la Dirección Nacional de Educación Media emitió una Circular ( $\left.n^{\circ} 206\right)$ en la que se establecieron algunas especificaciones en relación a la separación y expulsión de los alumnos. 
Allí se indicaba que diversos "defectos formales" del Reglamento daban lugar a reclamos, por vía de recursos administrativos, que debían ser acogidos en detrimento del principio de autoridad de la dirección de los establecimientos. Por otra parte, señalaba la necesidad de que las sanciones se ajustasen a las normas, cuidando la legitimidad de las medidas y evitando toda "sensación de arbitrariedad". La Circular establecía también que las medidas disciplinarias tenían que ser notificadas de inmediato y fehacientemente al alumno y a su padre, tutor o encargado. En el caso de que éstos no asistieran al establecimiento, debía quedar asentado para "dejar debidamente acreditado que se ha posibilitado ejercer el derecho de defensa". Acorde al espíritu democrático, la circular se preocupaba por recalcar que se debía dejar sentado, por escrito, que el alumno había sido escuchado. Y si, luego de todo el proceso, se avanzaba en la separación temporal del alumno del establecimiento, el Rectorado o la Dirección debía dictar "resolución fundada", una vez constatada la falta. Asimismo, la normativa avanzaba en ajustar lo relativo a las faltas colectivas. Las sanciones no debían tener un espíritu injusto y, por ello, debía establecerse el grado de responsabilidad de cada uno de los partícipes. De ese modo, la medida disciplinaria podía ser graduada.

Como puede observarse, las primeras transformaciones normativas se orientaron a otorgar una mayor legitimidad y fundamentación a los procedimientos disciplinarios, fenómeno que, desde nuestra perspectiva, debe ser comprendido a la luz de las luchas democráticas características del período. En relación con ello, y dentro del marco más general de debate educativo, el Informe sobre posibles reformas del sistema educativo, elaborado en 1987 por la Comisión Honoraria de Asesoramiento del Congreso Pedagógico, señalaba la necesidad de "democratizar" la educación y, por ende, el régimen disciplinar. En ese sentido, planteaba que el autoritarismo, representado por la figura del docente como "magíster dixit", aún subsistía. A ese modelo debía contraponérsele el de un "auténtico educador", que se constituiría en un guía, orientador y animador de los estudiantes. Asimismo, propiciaba una "educación participativa", es decir, una educación que debía promover la intervención activa de docentes, padres y alumnos en la gestión administrativa de los establecimientos.

En el acto de clausura del Congreso Pedagógico se retomaron algunos de los puntos anteriormente señalados. En 1988, una de las comisiones del Congreso (Comisión $n^{\circ} 5$ ) advertía que el autoritarismo era el modo de relación predominante en las escuelas. Por otra parte, la Comisión $n^{\circ} 7$, dedicada al examen de las formas de "Gobierno y financiamiento de la educación", detectaba otro problema: la falta de participación. En ese sentido, planteaba que una de las metas del sistema educativo debía ser acrecentar la intervención protagónica de todos los integrantes de la comunidad, especialmente de la familia, en la vida educativa, con igual responsabilidad social en todas las acciones que se realizaran. Este aspecto resulta importante ya que uno de los 
dictámenes de la Comisión, si bien no obtuvo consenso, avanzó en trazar una de las grandes líneas del cambio que se efectuaría en la próxima década: la creación de Consejos de escuela. No sólo ello. También se encargó de fijar cuáles debían ser sus características: tendrían un carácter consultivo, asesor, directivo o deliberativo, según la decisión que tomara cada comunidad educativa, de acuerdo a la situación jurídica del establecimiento y de los derechos adquiridos por los docentes.

Cabe destacar que esta perspectiva "participativa", característica del período, puede rastrearse también en diversas disposiciones implementadas en el sistema educativo como, por ejemplo, aquellas referidas a la creación de talleres de reflexión con la participación de docentes, padres y alumnos (Circ. $102,132 / 85,75,166 / 86$ ) y en la incorporación de nuevas tareas, además de las estrictamente económicas, en las cooperadoras escolares (Circ. 16/84, 134, 208/86, 28/87).

Como un momento de síntesis, hacia 1989, la Dirección General de Planificación Educativa confeccionó un informe titulado "La disciplina escolar. La normativa vigente y su aplicación". Allí, los especialistas indicaban que para que un código normativo fuera respetado, su significado debía ser conocido y aceptado por todos los miembros de la comunidad educativa. Al respecto, sostenían que podía existir un abismo entre, por ejemplo, lo conceptuado como falta de respeto por los representantes del mundo adulto y los adolescentes. Sin embargo, señalaba que el Reglamento de 1943 sólo especificaba las causas de aplicación de las sanciones en el caso de la expulsión e ignoraba aquellas que originaban una suspensión o amonestación. Asimismo, la enumeración de las causas de expulsión no necesariamente restringía posibles arbitrariedades. Desde su perspectiva, el modo escolar se encontraba caracterizado por el autoritarismo. El "modelo participativo" sería su opuesto, entendiendo por participación la posibilidad de amplios sectores de tener una intervención real en la toma de decisiones de la institución escolar. Por otro lado, sostenía que la conducta de los alumnos no debía ser más un prerrequisito para que el docente pudiera enseñar, sino que tenía que ser una resultante de la propuesta de enseñanza del docente y de aquella que apoyase la institución. Así, se iba proyectando que el cambio en el régimen disciplinar debía ir de la mano de un proceso de reforma más abarcativo.

Como hemos visto, a partir de la década del ' 80 comenzaron a surgir nuevas concepciones sobre la disciplina escolar, caracterizadas por tres elementos íntimamente vinculados: el rechazo del "autoritarismo", la búsqueda de un régimen disciplinario legítimo y la promoción de la participación del conjunto de la comunidad educativa en los asuntos escolares. En ese sentido, Inés Dussel y Myriam Southwell (2009) indican que, luego de la última dictadura militar, la reapertura democrática colocó a la autoridad en el lugar de aquello que había que eliminar. Desde nuestra perspectiva, en esos años comenzó a perfilarse 
cierto consenso en relación a que no sólo las autoridades sino también los alumnos y sus padres debían ser los encargados de solucionar las "situaciones conflictivas" en el espacio escolar. Por ello, consideramos que la transformación radical que supuso la creación del Sistema Escolar de Convivencia, hacia fines de los años '90, se fue configurando durante los diez años previos. De hecho, hacia 1989, el Ministerio de Educación nacional elaboró un Proyecto de Reglamento General para los Establecimientos de Nivel Medio mediante el cual se creaban consejos de escuela. En ellos, participarían los alumnos y sus familias. Sin embargo, esta iniciativa no logró cuajar a nivel nacional ya que la normativa no llegó a entrar en vigencia. Sin embargo, y retomando lo actuado, en la década del ' 90 veremos emerger una expresión de las tendencias señaladas en una jurisdicción en particular: la Ciudad de Buenos Aires. Veamos.

\section{El Sistema Escolar de Convivencia}

En el año 1996, mediante la Ordenanza n 51.284 , fue creado el Sistema de Convivencia Institucional en la Ciudad de Buenos Aires. Sin embargo, pocos meses después, el Gobierno de la Ciudad vetó aquella disposición. Años más tarde, el 5 de agosto de 1999, luego de un amplio debate parlamentario, la Legislatura sancionó la Ley $n^{\circ} 223$ que creó el Sistema Escolar de Convivencia. Dicho sistema es el conjunto de principios, normas, órganos y prácticas que regulan las relaciones entre los miembros de la comunidad educativa de cada institución. Entre sus objetivos se encuentra la participación democrática de todos los sectores de la comunidad educativa, de acuerdo a la competencia y responsabilidad de cada uno. Su participación incluye la elaboración de las normas que rigen la convivencia institucional, la búsqueda de consensos a través del diálogo y el proporcionar a las instituciones mecanismos para la resolución de los conflictos que se susciten, entre otros. En suma, el Sistema de Convivencia se organiza en cada escuela con la participación de toda la comunidad educativa.

Un paso previo a la instauración del nuevo sistema fue la eliminación de las amonestaciones mediante la Ordenanza $n^{\circ} 51.870$ de 1997. Luego, con la creación del Sistema Escolar de Convivencia, se pautó un nuevo criterio para las sanciones -que, además, dejaron de ser acumulativas. El nuevo sistema establecía, en orden de importancia, el siguiente esquema de faltas: apercibimiento oral, apercibimiento escrito, realización de acciones reparatorias en beneficio de la comunidad escolar, cambio de división, cambio de turno y separación del establecimiento. En este caso también se fijaba que todas las sanciones debían ser comunicadas fehacientemente a los alumnos y a sus responsables, indicando su causa y la fundamentación de la medida. En el caso de decidirse la medida más extrema, a saber, la separación del alumno del establecimiento, se encargaba a la Secretaría de Educación el garantizar la continuidad de los 
estudios del alumno sancionado en otro establecimiento. Se dejaba atrás, así, la pérdida del año escolar que ocasionaba el antiguo reglamento de 1943.

Por otra parte, la nueva normativa fijaba que en cada escuela estatal debía constituirse un Consejo Escolar de convivencia. Formarían parte del Consejo la Rectoría y representantes -elegidos mediante votación- de los profesores, preceptores, alumnos, padres, madres o tutores, asesores pedagógicos, psicólogos, psicopedagogos y del Centro de Estudiantes. La nueva normativa delegó al Consejo diversas funciones tales como dictar el reglamento interno de la institución, garantizar la difusión de las normas a toda la comunidad educativa y revisarlas anualmente, crear otros organismos de participación para la resolución de los conflictos, proponer sanciones ante las transgresiones de las normas remitidas a su consideración, elaborar formas de prevención de los problemas de convivencia, entre otras.

Recién dos años más tarde de su creación, el Sistema Escolar de Convivencia fue reglamentado mediante el Decreto $n^{\circ} 1.400$ de 2001. La nueva norma establecía, por un lado, que cada sanción aplicada requería de una instancia de reflexión conjunta sobre los comportamientos inadecuados. Además, para superar las situaciones de conflicto, debían adoptarse acciones que incluyeran el compromiso de las partes involucradas así como acciones conjuntas entre la escuela y la familia. El Decreto no eliminó como posibilidad la separación del alumno del establecimiento. Al respecto, fijó tres formas: temporal, por el resto del año escolar o definitiva. Esta última sólo se aplicaría de existir, en forma cierta o inminente, un daño grave a la integridad física, psíquica o moral de los integrantes de la comunidad educativa o a la propiedad y bienes de la institución y/o de sus integrantes, o bien una inconducta grave o reiterada que no pudiera solucionarse mediante la reflexión o reparación. En el caso de la separación transitoria o por el resto del año escolar se contemplaba la reubicación del alumno en otro establecimiento. Por su parte, las solicitudes de cambio de turno y separación del establecimiento debían ser aplicadas por el Director o Rector, previa intervención del Consejo Escolar. Por otro lado, las normas internas desarrolladas por el Consejo serían aprobadas por el Rectorado o Dirección. A su vez, éstos debían buscar formas de resolución de los conflictos evitando la aplicación de sanciones.

A la hora de interpretar los cambios que la Ciudad puso en marcha en su régimen disciplinar, Mariano Narodowski (1999) señala que la nueva normativa se habría ajustado íntegramente a las tendencias tradicionales de la política educativa argentina: la hiperregulación de las escuelas públicas y la desregulación de las escuelas privadas. Desde su perspectiva, dos aspectos del Sistema Escolar de Convivencia darían cuenta de esa afirmación. Por un lado, la participación directa del Estado en la definición de cuestiones que serían estrictamente pedagógicas, normativizando la vida cotidiana de los estableci- 
mientos educativos. Por el otro, el hecho de que los Consejos de Convivencia son optativos para las escuelas privadas.

Narodowski considera que la obligatoriedad de los Consejos Escolares una respuesta única frente a problemas de índole diversa y heterogénea. Desde su perspectiva, esta "rigidez" en las intervenciones sobre el conflicto escolar podría contribuir a incrementar, o a no detener, la deserción y el "fracaso escolar" de quienes tienen un punto de partida más desventajoso en relación a las culturas escolares dominantes.

Desde nuestra perspectiva, de manera contraria a lo señalado por Narodowski, la instauración del Sistema Escolar de Convivencia supuso una completa fragmentación del régimen disciplinario escolar. Tanto la tipificación de los actos de indisciplina como los mecanismos de sanción se tornaron cada vez más difusos, dando lugar a tantos criterios como Consejos Escolares existieran. En ese sentido, Inés Dussel (2005) observa, correctamente, que con la sanción del Sistema Escolar de Convivencia la ley escolar se tornó más ambigua. Desde su perspectiva, esa ambivalencia se encontraría en que los reglamentos de convivencia combinan temas y estrategias nuevos y, al mismo tiempo, viejos en relación a la disciplina.

Entendemos que existe otro aspecto que debe ponerse de relieve a la hora de analizar los Consejos de Convivencia. A nuestro juicio, la creación de los consejos escolares y la eliminación de las amonestaciones, al suponer una flexibilización del régimen disciplinario -al menos, en términos comparativos con el Reglamento de 1943- coadyuvan a frenar la deserción escolar al eliminar uno de los motivos que podrían desencadenar la salida del sistema. En ese sentido, los Consejos parecieran indicar una búsqueda de retención de los alumnos en el espacio escolar. Así las cosas, no llama la atención que el Ministerio de Educación de la Nación propusiera en el 2009 la extensión, a todo el país, de los Consejos de Convivencia como un instrumento para garantizar la permanencia y el egreso de todos los alumnos del nuevo secundario obligatorio. Curiosamente, ese proyecto tiene lugar al mismo tiempo que la ciudad pionera en desarrollar aquella experiencia, frente a numerosos episodios de violencia en las escuelas, se encamina a reestructurar su sistema disciplinar.

En ese sentido, en el año 2008, mediante el Decreto $n^{\circ} 998$, fue sustituida la anterior reglamentación del Sistema Escolar de Convivencia de la Ciudad (Decreto 1.400/01). Por un lado, si antes no habían sido pautadas las conductas contrarias al sistema de convivencia, ahora el artículo $6^{\circ}$ establecía que:

Resultan especialmente contrarias al Sistema Escolar de Convivencia y al espíritu democrático, e inadmisibles en la educación pública de la Ciudad Autónoma de Buenos Aires, y por ende, pasibles de sanción, sin perjuicio de las que correspondan a otras faltas que pudieren cometerse, las siguientes conductas: agresión física o verbal; ofensa a los símbolos patrios y/o religiosos; ofensas motivadas en 
cuestiones étnicas, de género, nacionalidad u orientación sexual; daños al patrimonio escolar; permanecer en el establecimiento escolar fuera del horario de clase o retirarse de él, en ambos casos sin la autorización correspondiente.

El último punto es especialmente relevante en tanto fija la posibilidad de establecer sanciones en el caso de tomas de colegios.

Asu vez, el nuevo Decreto estableció que las sanciones debían ser acumulativas, quedando a criterio del Director o Rector la decisión de prescindir de este principio en el caso de que las circunstancias -excepcionales- lo aconsejaran.

En relación a los miembros del Consejo Escolar, se establecieron diversas condiciones para acceder en calidad de tal. Los docentes deben acreditar, como mínimo, un año de antigüedad. Los alumnos e integrantes del Centro de Estudiantes no pueden presentar antecedentes disciplinarios desfavorables y, al mismo tiempo, deben acreditar un muy buen rendimiento académico. Cabe destacar que este punto, sumado a los límites que se buscan establecer a las tomas de colegios, cobra suma importancia en el contexto actual, caracterizado por una ascenso de los reclamos y luchas de los estudiantes secundarios. Por su parte, padres, tutores y/o encargados pueden ser miembros del Consejo siempre y cuando los alumnos a quienes representan hubieran sido matriculados en el establecimiento durante, por lo menos, dos años. Por último, alumnos, padres y representantes del Centro de Estudiantes pueden ser elegidos a partir del segundo año del nivel.

Por otro lado, la normativa reforzó, mediante diversas disposiciones, la potestad de la máxima autoridad de los establecimientos escolares. Entre ellas se encuentra la creación de los "Consejos de Emergencia". Estos nuevos consejos, integrados por el Director o Rector, un representante de profesores, otro de alumnos y un tercero de padres, son presididos por los directivos, quienes, a su vez, tienen la facultad de convocar a los miembros cuando lo consideren necesario. Cabe aclarar que dichos miembros pueden no pertenecer al Consejo de Convivencia de la escuela.

Desde diversos ángulos del espectro político se ha sostenido que la nueva reglamentación es una avanzada contra la democratización escolar. Sin embargo, lo cierto es que los elementos centrales del Sistema Escolar de Convivencia no han sido alterados.

\section{Conclusiones}

A partir de lo expuesto, pueden establecerse tres grandes momentos 0 etapas del régimen de disciplina correspondiente al nivel medio. El primero, desde la sanción del Reglamento General para los establecimientos de enseñanza secundaria, normal y especial en 1943, hasta principios de los años '80, estuvo caracterizado por un régimen disciplinario prescriptivo y centrado en la 
estructura jerárquica de las autoridades educativas. Este sistema se mantuvo prácticamente inalterado hasta la década del ' 80.

Como hemos visto, durante la última dictadura militar la disciplina fue reforzada de hecho y orientada según las tareas políticas que se propuso realizar. Una de ellas consistió en eliminar al movimiento estudiantil. Ahora bien, las herramientas legales para llevarla a cabo ya estaban dadas, en el caso del nivel medio, desde la sanción del Reglamento de 1943. Asimismo, una parte medular de aquella tarea fue desarrollada en forma clandestina. Por otra parte, la búsqueda de "despolitización" de las escuelas secundarias fue de la mano de un aumento de la exigencia en los rendimientos escolares de los estudiantes. Dicho fenómeno se expresó, por ejemplo, en el aumento de las exigencias del sistema de evaluación. A su vez, la dictadura buscó asegurar la disciplina a partir de aquel sistema. De esa forma, los docentes debían evaluar, de manera permanente, las conductas de los estudiantes. Así, ambos aspectos de la realidad educativa -disciplina y evaluación- estrecharon filas. El docente debió convertirse en el guardián del orden -político- y calificarlo.

Por las razones expuestas anteriormente, sostendremos que no resultó apremiante para el régimen dictatorial modificar el reglamento que regulaba la disciplina escolar en el nivel medio desde principios de la década del ' 40 .

Por otra parte, hemos visto cómo la educación en el patriotismo cobró renovada importancia en lo que refiere al ámbito disciplinario. Desde nuestra perspectiva, la ideología que plantea como realidad primaria el hecho nacional intenta desdibujar las contradicciones existentes en una sociedad dividida en clases sociales, estableciendo la igualdad ilusoria de sus miembros, los "ciudadanos" (Sartelli, 1996). Por ello, resulta clave en los procesos de reconstrucción de la hegemonía burguesa, objetivo central del régimen castrense.

En un segundo momento, desde la reapertura democrática en 1983 hasta mediados de la década del '90, comenzó a establecerse la argamasa ideológica que dará lugar, en la etapa posterior, a la instauración del Sistema de Convivencia. Como hemos dicho, sus notas centrales fueron el establecimiento de ciertos resguardos en relación a la arbitrariedad que podrían presentar las decisiones adoptadas por las autoridades educativas y la promoción de la participación de la comunidad educativa en su conjunto en diversas esferas de actividad de las instituciones educativas.

Por último, una tercera etapa se inauguró con la creación del Sistema Escolar de Convivencia. En un primer momento, en una jurisdicción en particular (Capital Federal). Sin embargo, en tanto aquella experiencia expresaba cierto consenso, más general, en torno a la necesidad de un nuevo sistema disciplinario, más "participativo" y "democrático", con el paso del tiempo diversas jurisdicciones comenzarían a desarrollar procesos de reforma similares.

Ahora bien, en un contexto de crisis económica y social cada vez más aguda, nos preguntamos qué implicancias tiene el componente central del 
sistema de convivencia: el deber dialogar y consensuar frente a cada situación conflictiva. Sostendremos como hipótesis que las reformas en el régimen disciplinario, al igual que lo ocurrido en el régimen de evaluación, expresan una necesidad política de retener a los jóvenes dentro de las escuelas. En ese sentido, resultan ilustrativos algunos de los fundamentos del Proyecto de Ley de Creación del Sistema Nacional de Convivencia Escolar presentado ante la H. Cámara de Diputados de la Nación (2009):

La extensión de los estudios secundarios y su obligatoriedad si bien significan un avance, es un desafío todavía a lograr, teniendo en cuenta que la universalización de la escolaridad primaria se logró después de décadas en el país, alcanzando hoy una inserción educativa del $98 \%$. Sería deseable acotar los tiempos para el logro de esa meta correspondiente a la educación secundaria, sin que debamos soslayar la importancia de la inclusión y la participación colegiada de nuestros jóvenes en el sistema educativo. Para ello debemos debatir ampliamente aspectos que alcanzan al régimen escolar disciplinario que hoy tiene vigencia en el sistema educativo secundario. (p. 6)

Como vemos, el proyecto propone, de manera explícita, revisar el sistema disciplinario en pos de frenar la deserción escolar en el nivel secundario.

Durante los últimos años han comenzado a escucharse numerosas voces pidiendo el retorno de las amonestaciones y/o la eliminación de los Consejos Escolares. Sin embargo, por ser muy recientes, tal vez debamos esperar algún tiempo más para poder afirmar si nos encontramos, o no, ante una nueva etapa. Por otra parte, como hemos visto existen propuestas contrarias, orientadas a extender el Sistema Escolar de Convivencia al conjunto del país.

Por último, quisiéramos destacar que las transformaciones observadas en el régimen de disciplina han sido llevadas a cabo por gobiernos de distinto signo político. Desde nuestra perspectiva, ello implica que detrás de las intenciones proclamadas por unos y otros existe una estrategia de clase. Haciéndose eco de las demandas de democratización educativa, la burguesía ha logrado que se desdibuje una de sus estrategias en materia educativa, orientada a contener una parte del conflicto social dentro de los muros escolares: la disminución progresiva de las exigencias y requisitos disciplinares.

\section{Referencias Bibliográficas}

- Bruera, R. P. (1976). Palabras pronunciadas por el Ministro de Educación al asumir su cargo. Buenos Aires: Ministerio de Cultura y Educación.

- Comisión Honoraria de Asesoramiento del Congreso Pedagógico Nacional (1987). Informe sobre posibles reformas del sistema educativo. Buenos Aires: Ministerio de Educación y Justicia. 
- Dirección General de Instrucción Pública (1943). Nota de elevación del proyecto de Reglamento para los establecimientos de Enseñanza Media. Buenos Aires: Ministerio de Justicia e Instrucción Pública.

- Dirección General de Enseñanza Secundaria, Normal, Especial y Superior (1957). Reglamento General para los establecimientos de enseñanza secundaria, normal y especial (texto ordenado). Buenos Aires: Ministerio de Educación y Justicia.

- Dirección General de Planificación Educativa (1989). La disciplina escolar. La normativa vigente y su aplicación, Buenos Aires: Ministerio de Educación y Justicia.

- Dussel, I. (2005). ¿Se renueva el orden disciplinario escolar? Una lectura de los reglamentos de convivencia en la Argentina de la post-crisis? Revista Mexicana de Investigación Educativa, 10, 1109-1121.

- Dussel, I. y Southwell, M. (2009). La autoridad docente en cuestión: líneas para el debate. El monitor de la educación, 20, 26-28.

- H. Cámara de Diputados de la Nación (2009). Proyecto de Ley de Creación del Sistema Nacional de Convivencia Escolar en los establecimientos educativos secundarios, Expediente 4299-D. Buenos Aires: Imprenta del Congreso de la Nación.

- Ministerio de Educación y Justicia (1988). Informe Final del II Congreso Pedagógico Nacional. Buenos Aires.

- Ministerio de Cultura y Educación (1978). Boletín de Comunicaciones $N^{\circ}$ 61, Buenos Aires.

- Ministerio de Educación y Justicia (1989). Proyecto de Reglamento General para los establecimientos de nivel medio. Buenos Aires.

- Narodowski, M. (1999). Especulación y castigo en la escuela secundaria. Tandil: Universidad Nacional del Centro.

- $\quad$ Pineau, P. (2006). El principio del fin. Políticas y memoria de la educación en la última dictadura militar (1976-1983). Buenos Aires: Colihue.

- Sartelli, E. (1996). Celeste, blanco y rojo. Democracia, nacionalismo y clase obrera en la crisis hegemónica. Revista Razón y Revolución, 2, 1-60. Extraído el 31 de Agosto de 2010 desde http://www.razonyrevolucion.org.ar/textos/revryr/luchadeclases/ ryr2SartelliCeleste.pdf

- Sartelli, E. (2007). La plaza es nuestra. Buenos Aires: Ediciones ryr. 